\title{
Effect of Nursing Teaching Guideline on Patient Anxiety before Radical Nephrectomy at Assiut University Hospital
}

\author{
Hanan Ali Ahmed', Zienab Abd El-lateef Mohammad², Esmat Sayed Abdelmaged ${ }^{3}$, Ahmed Abdelhamid \\ Shahat $^{4}$ \& Medhat Ahmed Abdalla ${ }^{5}$. \\ 1. Assistant lecturer of Medical-Surgical Nursing, Faculty of Nursing, Assiut University, Egypt. \\ 2. Professor of Medical-Surgical Nursing, Faculty of Nursing, Assiut University, Egypt. \\ 3. Assistant Professor of Medical-Surgical Nursing, Faculty of Nursing, Assiut University, Egypt. \\ 4. Assistant Professor of Urology, Faculty of Medicine, Assiut University, Egypt. \\ 5. Professor of Urology, Faculty of Medicine, Assiut University, Egypt.
}

\begin{abstract}
Background: Radical nephrectomy is the standard treatment of choice for localized renal cell carcinoma. Ensuring that patients' informational needs have been met before radical nephrectomy set the stage for successful management and improves recovery. Aims: Evaluate the effect of nursing teaching guideline on the level of anxiety before radical nephrectomy. Research design: True experimental research design (Pre-test and post-test). Setting: Assiut Urology and Nephrology Hospital. Patients: Sixty adult patients undergoing radical nephrectomy were distributed into two equal groups (study and control groups) 30 patients for each. Tools: (I) Patient assessment sheet, (II) State-Trait Anxiety Inventory for Adult, and (III) The designed nursing teaching guideline for patients undergoing radical nephrectomy. Results: Statistically significant differences were found between study and control groups regarding preoperative anxiety levels $(\mathrm{P}<0.01)$. Conclusion: The levels of preoperative anxiety significantly reduce associated with preoperative nursing teaching guideline provision.
\end{abstract}

\section{Keywords: Radical Nephrectomy \& Nursing Teaching Guideline.}

\section{Introduction}

Surgical resection of renal cell carcinoma (RCC) is the standard for long-term cure of the disease. Radical nephrectomy $(\mathrm{RN})$ is a safe standard procedure and has demonstrated reliable oncological control. On the other hand, surgeons need to consider that the majority of individuals with localized RCC will survive their cancer and therefore every effort has to be made to minimize treatment related morbidity (Mirza et al., 2016 \& Vukovic \& Dinic, 2018).

The experience of stress in patients with RCC through helplessness and the suppression of emotions correlate with unfavorable disease prognosis and associated with increased suicide rate among patients with renal tumor. Surgical treatments are never without risks and adverse events. Patients with renal tumors and patients undergoing $\mathrm{RN}$ especially females presented higher levels of anxiety (Pastore et al., 2017 \& Ryamukuru, 2017).

Preoperative anxiety is the most common problem in the preoperative care of patients. It can be seen as a complex organic reaction that happens only in situations considered to be dangerous. Intense anxiety experienced in the preoperative period increases the intensity of pain in the postoperative period and makes pain control difficult. The patient feels anxiety as a result of the physical effects caused by the disease as well by the change of environment imposed by hospitalization. The goal of perioperative nursing care is to provide better environments and decrease anxiety of the patient before, during and after surgery (Karabulut \& Cetinkaya, 2011 \& Alper \& Yüksel, 2016).

Also there are other factors that constitute significant sources of anxiety including anesthesia, operation, complications, recurrence following surgical treatment and some invasive interventions that raise real and unreal fears. For the patient, an operation implies pain, distortion of body image, loss of independence and physical separation from family (Pinto et al., 2016 \& Draeger et al., 2018).

The degree to which each patient manifests anxiety depends on many factors such as age, gender, educational status, past experiences with the surgery, type and extent of the proposed surgery, current health status, and socioeconomic status. Identifying risk factors helps the nurse to provide psychological support during the preoperative period so that stress can be decreased lead to anxiety decrease (Mulugeta et al., 2018).

Anxieties of patients in general are found with lack of information. Then education reduces anxiety, preoperative education and informing is the first step in the psychological preparation of patients. These feelings can have negative effects on patients' health status. Knowledge about their condition and surgical and anesthetic procedures can help to reduce their anxiety levels (Palese et al., 2011 \& Harris et al., 2015). 
The information provided to patients before surgery, respect and empathy of nurses towards the patients and openness of nurses to the patients contribute positively to alleviate anxiety level of the patients awaiting surgery. This knowledge qualifies nurses to manage successfully the patient anxiety without medical interventions. Successful preoperative anxiety management may result in quick recovery, fewer complications, short hospital stay and less postoperative analgesic demands (Komolafe et al., 2015).

\section{Significance of the study}

According to researcher's experience; patients undergoing RN suffer from high level of preoperative anxiety and they don't receive enough information regarding their conditions. So, this study will be conducted to provide nursing teaching guidelines for those patients about $\mathrm{RN}$ to improve their knowledge and reduce preoperative anxiety.

\section{Aim of the study}

To evaluate the effect of nursing teaching guideline on patient anxiety before radical nephrectomy.

\section{Research hypothesis}

To fulfill the aim of the study, the following hypothesis was formulated preoperative anxiety level for the study group is supposed to be less than the control group.

\section{Patients \& Methods}

\section{Research design}

Prospective randomized controlled trial research design was utilized in this study. The researcher put 60 numbered small piece of paper into a package (160) (this is the number of sample). Ask patient to choose one piece of paper from the package without looking (this is patient's number). Single numbers was included in the control group while double numbers was included in the study group.

\section{Variables}

The independent variable was the nursing teaching guidelines while the dependent variables were preoperative anxiety level.

\section{Setting}

The study was conducted at Assiut Urology and Nephrology University Hospital.

\section{Sample}

Sixty adult patients undergoing RN were included in the study with the following criteria; age ranged from 18 - 65 years from both sexes. Patients were divided into two groups (study and control groups), 30 patients for each. Study group patient received the nursing teaching guidelines while the control group patients will receive routine hospital care. Patients undergoing laparoscopic radical nephrectomy were excluded.

Tools

The following tools were utilized for data collection:

Tool I: Patient assessment sheet

It was developed by the researcher after current national and international literature review. It consisted of three parts:

Part I: Demographic data about the patients: Name, age, sex, occupation, level of education, marital status and residence.

Part II: Medical data: Past and present health history, duration of illness, criteria of tumor, stage of RCC, medical treatment, patient's habits, laboratory investigations, vital signs and chronic disease.

Part III: Factors that may be responsible for preoperative anxiety among patients: Fear of complications, result of operation, concern about family, fear for one's life, postoperative pain, awareness during surgery, fear of disability, need of blood transfusion, waiting for operation, financial loss, fear of unknown, getting stuck with needle, harm from doctor/ nurse mistake, and fear of death.

Tool II: State-Trait Anxiety Inventory for Adults \{State form\}

It was developed by Spielberger et al, (1983). It is the definitive instrument for measuring anxiety in adults. It was adapted by the researcher to assess preoperative. It includes 20 items to assess level of anxiety. All items are rated on a 4-point scale $(1=$ not at all, $2=$ somewhat, $3=$ moderately so, $4=$ very much so). Scores range from 20 to 80 . The scores of $(0<20$ no anxiety, $20<40$ normal anxiety, $40<60$ above the moderate level of anxiety, and $60-80$ severe anxiety).

Tool III: The designed nursing teaching guidelines for patients undergoing radical nephrectomy:

The nursing teaching guidelines were developed by the researcher after reviewing current national and international literatures. The guideline included:

- Definition of RN.

- Brief description for surgery (radical nephrectomy).

- Complications of RN.

- Nursing guidelines for patients undergoing RN:

- Nursing care and teaching before, during and after surgery.

- Medications.

- Wound care.

- Diet.

- Stress reduction.

- Physical activity and exercise.

- Smoking cessation. 
- Routine follow up and when it is necessary to seek medical help and immediately go to the hospital.

\section{Ethical approval}

Permission to carry out the study was obtained from the ethical committee of the Faculty of Nursing. An official letter was issued from the Dean of the Faculty of Nursing to the Head of the Urology Department to collect the necessary data, and explain the aim and contents of the study to nursing supervisors and surgeons to gain their cooperation. Informed consent was obtained from patients or families that are willing to participate in the study after explanation of the nature and purposes of the study. Confidentiality and anonymity assured. Patients have the right to refuse to participate and or withdraw from the study without any rational at any time.

\section{Procedure}

The study was carried out on 3 phases

The first phase (preparatory phase)

Preparation of the data collection tools and the designed nursing teaching guidelines were carried out by the researcher after extensive literature review (nursing and medical textbooks, journals and internet resources), this phase ended by content validity and pilot study.

\section{Content validity}

It was checked and revised by special five expertise's ( 2 from the Medical staff of Urology and 3 from Medical - Surgical Nursing staff) who reviewed the tools for clarity, relevance, and applicability. Modification was carried out accordingly. Test reliability of the tools was ascertained with Cronbachs alpha (0.73) which showed that the internal consistency of the tools was good.

\section{Pilot study}

It was conducted on $10 \%$ of sample (6 patients) in the selected setting for testing clarity and applicability of the study tools. The purpose of the pilot study was to detect any particular problem in the statements, clarity, feasibility, and applicability of the tool. The data obtained from the pilot study were analyzed; no change was done, so the $10 \%$ of sample selected for the pilot study were included in the main study.

The second phase (Implementation phase):

- During preoperative period the researcher interviewed with both study and control groups the researcher introduces herself to initiate communication, explain the nature and purpose of the study. Base line data were established using tool (I).

- Patients who constituted the control group were exposed to routine hospital care received. The study group was given the designed nursing teaching guidelines by the researcher (tool III).
- The program contain one session which took about 40 - 50 minutes. The study was carried out in the morning and after non shifts. It gire for each patient individually.

- The nursing teaching guidelines were given on an individual basis and one of the family members was present to ensure patient support.

- During the session each patient was given a written copy of the guidelines (booklet) in clear Arabic language.

- A brief review from the patient to assess his or her understanding, then the researcher clarified any points that the patient didn't understand.

- The researcher ensured commitment of the study group patients to implement the nursing guidelines through daily visiting then during hospitalization.

- Data were collected through the period from the beginning of February 2018 to the end of May 2019.

\section{The third phase (Evaluation phase)}

Both study and control groups were evaluated preoperatively to evaluate the effect of the nursing teaching guidelines on preoperative anxiety by using tool (II).

\section{Statistical analysis}

Data were collected and statistical analysis was done using IBM SPSS 19 ®. Data expressed as mean, standard deviation, number and percentage. Quantitative data was analyzed using independentsamples t-test, while qualitative data was analyzed using Pearson chi square test. Probability level of < 0.05 was adopted as a level of significance for testing the research hypotheses. 


\section{Results}

Table (1): Distribution of demographic characteristics of patients (study and control groups).

\begin{tabular}{|c|c|c|c|c|c|c|c|}
\hline \multirow{3}{*}{ Age (years) } & \multicolumn{2}{|c|}{$\begin{array}{c}\text { Study group } \\
(\text { No. }=30)\end{array}$} & \multicolumn{2}{|c|}{$\begin{array}{c}\text { Control group } \\
(\text { No. }=30)\end{array}$} & \multicolumn{2}{|c|}{$\begin{array}{c}\text { Total } \\
(\text { No. }=60)\end{array}$} & P-value \\
\hline & & & \multirow{2}{*}{\multicolumn{2}{|c|}{$\frac{\text { Mean } \pm \text { SD }}{48.23 \pm 13.72}$}} & & & \multirow{3}{*}{$0.032^{*}$} \\
\hline & \multicolumn{2}{|c|}{$54.80 \pm 8.93$} & & & \multicolumn{2}{|c|}{$51.52 \pm 11.95$} & \\
\hline Gender & No & $\%$ & No & $\%$ & No & $\%$ & \\
\hline $\begin{array}{l}\text { - Male } \\
\text { - Female }\end{array}$ & $\begin{array}{l}17 \\
13\end{array}$ & $\begin{array}{l}56.7 \\
43.3\end{array}$ & $\begin{array}{l}16 \\
14\end{array}$ & $\begin{array}{l}53.3 \\
46.7\end{array}$ & $\begin{array}{l}33 \\
27\end{array}$ & $\begin{array}{l}55.0 \\
45.0\end{array}$ & $0.500^{\mathrm{Ns}}$ \\
\hline $\begin{array}{l}\text { Educational level } \\
\text { - High education } \\
\text { - Secondary } \\
\text { - Basic education } \\
\text { - Read and write } \\
\text { - Non educated }\end{array}$ & $\begin{array}{l}3 \\
6 \\
3 \\
3 \\
15\end{array}$ & $\begin{array}{l}10.0 \\
20.0 \\
10.0 \\
10.0 \\
50.0\end{array}$ & $\begin{array}{l}1 \\
5 \\
2 \\
8 \\
14\end{array}$ & \begin{tabular}{l|}
3.3 \\
16.7 \\
6.7 \\
26.7 \\
46.7
\end{tabular} & $\begin{array}{l}4 \\
11 \\
5 \\
11 \\
29\end{array}$ & \begin{tabular}{l|}
6.7 \\
18.3 \\
8.3 \\
18.3 \\
48.3
\end{tabular} & $0.463^{\mathrm{Ns}}$ \\
\hline $\begin{array}{l}\text { Occupation } \\
\text { - Office work } \\
\text { - Manual worker } \\
\text { - Not working } \\
\end{array}$ & \begin{tabular}{|l|}
5 \\
15 \\
10 \\
\end{tabular} & $\begin{array}{l}16.7 \\
50.0 \\
33.3 \\
\end{array}$ & $\begin{array}{l}1 \\
16 \\
13 \\
\end{array}$ & $\begin{array}{l}3.3 \\
53.3 \\
43.4 \\
\end{array}$ & $\begin{array}{l}6 \\
31 \\
23 \\
\end{array}$ & $\begin{array}{l}10.0 \\
51.7 \\
38.3 \\
\end{array}$ & $0.213^{\mathrm{Ns}}$ \\
\hline $\begin{array}{l}\text { Marital status } \\
\text { - Single } \\
\text { - Married } \\
\text { - Widow/ widower }\end{array}$ & $\begin{array}{l}- \\
25 \\
5\end{array}$ & $\begin{array}{l}- \\
83.3 \\
16.7\end{array}$ & $\begin{array}{l}3 \\
22 \\
5\end{array}$ & $\begin{array}{l}10.0 \\
73.3 \\
16.7\end{array}$ & $\begin{array}{l}3 \\
47 \\
10\end{array}$ & $\begin{array}{l}5.0 \\
78.3 \\
16.7\end{array}$ & $0.203^{\mathrm{Ns}}$ \\
\hline $\begin{array}{l}\text { Residence } \\
\text { - Urban } \\
\text { - Rural }\end{array}$ & $\begin{array}{l}16 \\
14\end{array}$ & $\begin{array}{l}53.3 \\
46.7\end{array}$ & $\begin{array}{l}10 \\
20 \\
\end{array}$ & $\begin{array}{l}33.3 \\
66.7 \\
\end{array}$ & $\begin{array}{l}26 \\
34 \\
\end{array}$ & $\begin{array}{l}43.3 \\
56.7 \\
\end{array}$ & $0.118^{\mathrm{Ns}}$ \\
\hline $\begin{array}{l}\text { Smoking } \\
\text { - Yes } \\
\text { - No }\end{array}$ & $\begin{array}{l}19 \\
11\end{array}$ & $\begin{array}{l}63.3 \\
50.0\end{array}$ & $\begin{array}{l}15 \\
15\end{array}$ & $\begin{array}{l}50.0 \\
50.0\end{array}$ & $\begin{array}{l}34 \\
26\end{array}$ & $\begin{array}{l}56.7 \\
43.3\end{array}$ & $0.297^{\mathrm{Ns}}$ \\
\hline
\end{tabular}

Person Chi-Square tests and Independent samples t-test $\quad$ Ns: Not significant $\quad{ }^{*}:$ Significant $(P<0.05)$

Table (2): Distribution of patients` medical data (study and control groups).

\begin{tabular}{|c|c|c|c|c|c|c|c|}
\hline Medical data & \multicolumn{2}{|c|}{$\begin{array}{l}\text { Study group } \\
(\text { No. }=30)\end{array}$} & \multicolumn{2}{|c|}{$\begin{array}{c}\text { Control group } \\
(\text { No. }=30)\end{array}$} & \multicolumn{2}{|c|}{$\begin{array}{c}\text { Total } \\
(\text { No. }=60)\end{array}$} & P-value \\
\hline \multirow[b]{2}{*}{$\begin{array}{l}\text { Location of tumor } \\
\text { - Right } \\
\text { - Left }\end{array}$} & No & $\%$ & No & $\%$ & No & $\%$ & \multirow[b]{2}{*}{$0.61^{\mathrm{Ns}}$} \\
\hline & $\begin{array}{l}15 \\
15\end{array}$ & $\begin{array}{l}50.0 \\
50.0\end{array}$ & $\begin{array}{l}13 \\
17\end{array}$ & $\begin{array}{l}43.3 \\
56.7\end{array}$ & $\begin{array}{l}28 \\
32\end{array}$ & $\begin{array}{l}46.7 \\
53.3\end{array}$ & \\
\hline $\begin{array}{ll}\text { Stage of renal cell carcinoma } \\
\text { - } & \text { Stage I: T1N0 M0 } \\
\text { - } & \text { Stage II: T2 N0 M0 } \\
- & \text { Stage III: T3 N1M0 }\end{array}$ & $\begin{array}{c}8 \\
16 \\
6\end{array}$ & $\begin{array}{l}26.7 \\
53.3 \\
20.0\end{array}$ & $\begin{array}{c}7 \\
12 \\
11\end{array}$ & $\begin{array}{l}23.3 \\
40.0 \\
36.7\end{array}$ & $\begin{array}{l}15 \\
28 \\
17\end{array}$ & $\begin{array}{l}25.0 \\
46.7 \\
28.3\end{array}$ & $0.34^{\mathrm{Ns}}$ \\
\hline $\begin{array}{ll}\text { Medical co-morbidities } \\
\text { - } & \text { Diabetes mellitus } \\
\text { - } & \text { Hypertension } \\
\text { - } & \text { Ischemic heart disease } \\
- & \text { Hepatitis } \\
\end{array}$ & $\begin{array}{l}5 \\
5 \\
- \\
1\end{array}$ & $\begin{array}{l}16.7 \\
16.7 \\
- \\
1.3 \\
\end{array}$ & $\begin{array}{l}- \\
9 \\
2 \\
2 \\
\end{array}$ & $\begin{array}{l}- \\
30.0 \\
6.7 \\
6.7 \\
\end{array}$ & $\begin{array}{l}5 \\
14 \\
2 \\
3 \\
\end{array}$ & $\begin{array}{l}8.3 \\
23.3 \\
3.3 \\
5.0 \\
\end{array}$ & \multirow[t]{2}{*}{$0.41^{\mathrm{Ns}}$} \\
\hline $\begin{array}{l}\text { Total medical } \\
\text { co-morbidities }\end{array}$ & 8 & 26.7 & 11 & 36.7 & 19 & 31.6 & \\
\hline \multirow[t]{2}{*}{ Hemoglobin } & \multicolumn{6}{|c|}{ Mean \pm SD } & \multirow[b]{2}{*}{$0.06^{\mathrm{Ns}}$} \\
\hline & $12.09 \pm 0.90$ & & 2.60 & & 12.34 & .03 & \\
\hline
\end{tabular}

Person Chi-Square tests and Independent samples test

Ns: Not significant 
Table (3): Comparison between study and control groups regarding clinical presentations

\begin{tabular}{|l|c|c|c|c|c|c|c|}
\hline \multirow{2}{*}{ Clinical presentations } & \multicolumn{2}{|c|}{$\begin{array}{c}\text { Study group } \\
(\text { No. = 30) }\end{array}$} & \multicolumn{2}{c|}{$\begin{array}{c}\text { Control group } \\
\text { (No.= 30) }\end{array}$} & \multicolumn{2}{c|}{$\begin{array}{c}\text { Total } \\
\text { (No. = 60) }\end{array}$} & \multirow{2}{*}{ P-value } \\
\hline Flank pain & No & \% & No & \% & No & \% & \multirow{2}{*}{0.573} \\
\cline { 2 - 8 } - Present & 22 & 73.3 & 20 & 66.7 & 42 & 70.0 & \\
- Not present & 8 & 26.7 & 10 & 33.3 & 18 & 30.0 & \\
\hline Gross hematuria & 16 & 53.3 & 12 & 40.0 & 28 & 46.7 & \multirow{2}{*}{0.301} \\
- Present & 14 & 46.7 & 18 & 60.0 & 32 & 53.3 & \\
- Not present & - & - & 5 & 16.7 & 5 & 8.3 & \multirow{2}{*}{0.06} \\
\hline Malaise & 30.0 & 100.0 & 25 & 83.3 & 55 & 91.7 & \\
\hline - Present & & & & & & \\
\hline
\end{tabular}

Person Chi-Square tests.

Table (4): Distribution of factors responsible for preoperative anxiety among patients (study and control groups).

\begin{tabular}{|c|c|c|c|c|c|}
\hline \multirow{3}{*}{$\begin{array}{l}\text { Factors } \\
\text { Fear of complications } \\
-\quad \text { Present } \\
-\quad \text { Not present } \\
\end{array}$} & \multicolumn{2}{|c|}{$\begin{array}{c}\text { Study group } \\
(\text { No. = 30) }\end{array}$} & \multicolumn{2}{|c|}{$\begin{array}{c}\text { Control group } \\
(\text { No. }=30)\end{array}$} & \multirow{3}{*}{$\begin{array}{r}\text { P-value } \\
0.519^{\mathrm{Ns}} \\
\end{array}$} \\
\hline & No & $\%$ & No & $\%$ & \\
\hline & $\begin{array}{c}23 \\
7\end{array}$ & $\begin{array}{l}76.7 \\
23.3\end{array}$ & $\begin{array}{c}25 \\
5\end{array}$ & $\begin{array}{l}83.3 \\
16.7\end{array}$ & \\
\hline $\begin{array}{ll}\text { Result of operation } \\
-\quad \text { Present } \\
-\quad \text { Not present }\end{array}$ & $\begin{array}{c}23 \\
7 \\
\end{array}$ & $\begin{array}{l}76.7 \\
23.3 \\
\end{array}$ & $\begin{array}{c}30.0 \\
0\end{array}$ & $\begin{array}{c}100.0 \\
0.0\end{array}$ & $0.005^{* * *}$ \\
\hline $\begin{array}{l}\text { Postoperative pain } \\
-\quad \text { Present } \\
-\quad \text { Not present }\end{array}$ & $\begin{array}{c}0 \\
30.0\end{array}$ & $\begin{array}{c}0.0 \\
100.0\end{array}$ & $\begin{array}{c}4 \\
26\end{array}$ & $\begin{array}{l}13.3 \\
86.7 \\
\end{array}$ & $0.038^{*}$ \\
\hline $\begin{array}{l}\text { Fear of disability } \\
-\quad \text { Present } \\
-\quad \text { Not present }\end{array}$ & $\begin{array}{c}2 \\
28\end{array}$ & $\begin{array}{c}6.7 \\
93.3\end{array}$ & $\begin{array}{c}0 \\
30.0\end{array}$ & $\begin{array}{c}0.0 \\
100.0\end{array}$ & $0.150^{\mathrm{Ns}}$ \\
\hline $\begin{array}{l}\text { Waiting for operation } \\
-\quad \text { Present } \\
-\quad \text { Not present }\end{array}$ & $\begin{array}{c}0 \\
30.0\end{array}$ & $\begin{array}{c}0.0 \\
100.0\end{array}$ & $\begin{array}{c}4 \\
26\end{array}$ & $\begin{array}{l}13.3 \\
86.7\end{array}$ & $0.038^{*}$ \\
\hline $\begin{array}{l}\text { Getting stuck with needle } \\
-\quad \text { Present } \\
-\quad \text { Not present }\end{array}$ & $\begin{array}{c}2 \\
28\end{array}$ & $\begin{array}{c}6.7 \\
93.3\end{array}$ & $\begin{array}{c}0 \\
30.0\end{array}$ & $\begin{array}{c}0.0 \\
100.0\end{array}$ & $0.150^{\mathrm{Ns}}$ \\
\hline $\begin{array}{l}\text { Fear of death } \\
\text { - Present } \\
\text { - Not present }\end{array}$ & $\begin{array}{c}0 \\
30.0\end{array}$ & $\begin{array}{c}0 \\
100.0\end{array}$ & $\begin{array}{l}15 \\
15\end{array}$ & $\begin{array}{l}50.0 \\
50.0\end{array}$ & $0.001^{* * *}$ \\
\hline
\end{tabular}




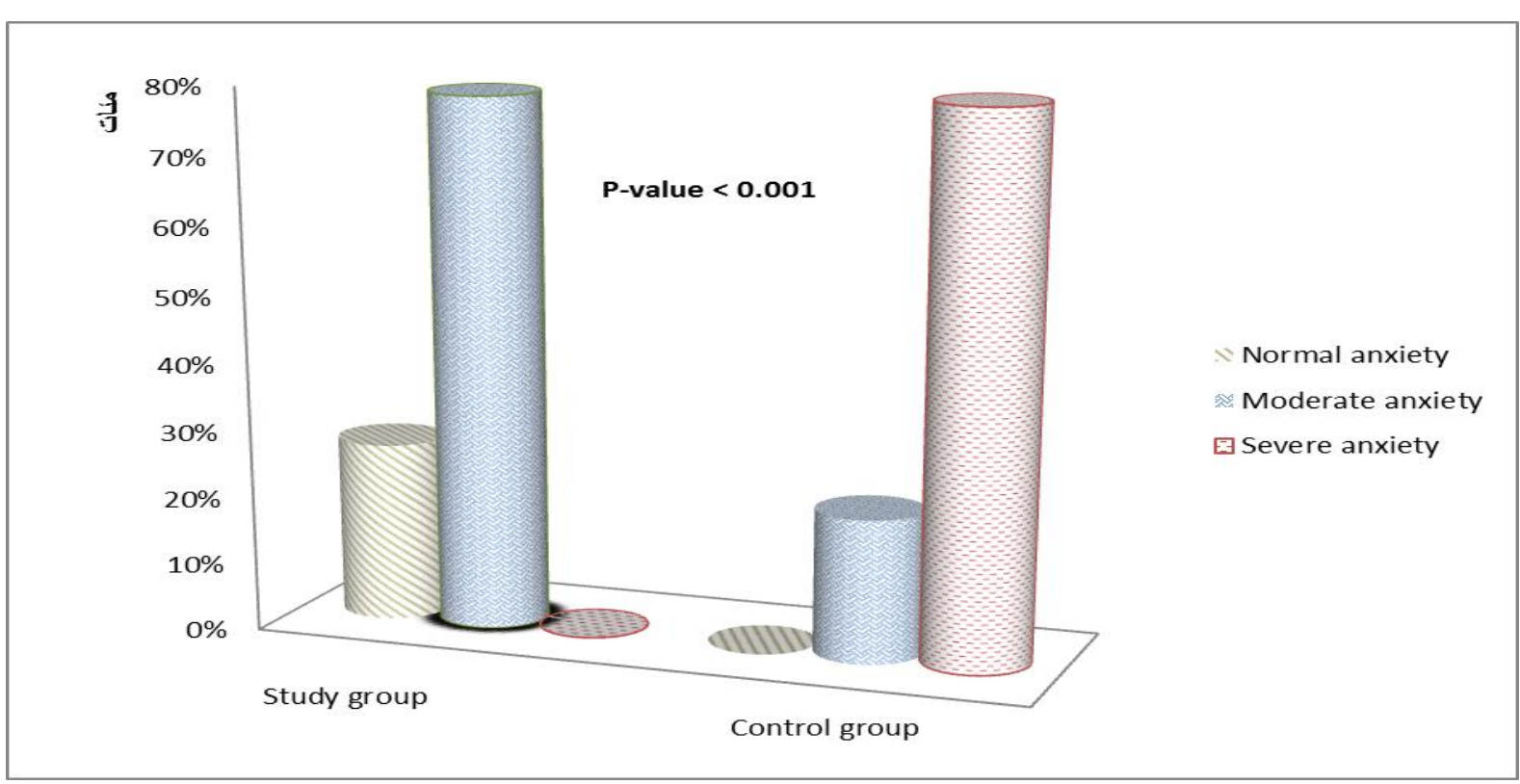

Fig (1): Comparison between the study and the control groups regarding preoperative anxiety.

Table (5): Relation between demographic data and preoperative anxiety among the studied sample $(\mathrm{n}=60)$.

\begin{tabular}{|c|c|c|c|c|c|c|c|c|c|c|c|c|c|}
\hline \multirow{3}{*}{ Factors } & \multirow{2}{*}{\multicolumn{6}{|c|}{$\frac{\text { Study group }(\text { No. }=30)}{\text { Level of anxiety }}$}} & \multicolumn{6}{|c|}{ Control group $($ No. $=30)$} & \multirow[t]{3}{*}{ P-value } \\
\hline & & & & & & & \multicolumn{6}{|c|}{ Level of anxiety } & \\
\hline & \multicolumn{4}{|c|}{ Moderate } & \multicolumn{2}{|c|}{ Severe } & & mal & & erate & \multicolumn{2}{|c|}{ Severe } & \\
\hline \multirow[t]{2}{*}{ Age (years) } & \multicolumn{6}{|c|}{ Mean \pm SD } & \multicolumn{6}{|c|}{ Mean \pm SD } & \multirow{2}{*}{$0.002^{* *}$} \\
\hline & \multicolumn{2}{|c|}{$53.3 \pm 6$} & \multicolumn{2}{|c|}{$55.3 \pm 9.8$} & \multicolumn{2}{|c|}{-} & & & \multicolumn{2}{|c|}{$61.3 \pm 5.7$} & \multicolumn{2}{|c|}{$44.9 \pm 13.2$} & \\
\hline \multirow{2}{*}{$\begin{array}{l}\text { Gender } \\
\text { - Male } \\
\text { - Female }\end{array}$} & No & $\%$ & No & $\%$ & No & $\%$ & No & $\%$ & No & $\%$ & No & $\%$ & \multirow[b]{2}{*}{$0.75^{\mathrm{Ns}}$} \\
\hline & $\begin{array}{l}5 \\
3\end{array}$ & $\begin{array}{r}16.7 \\
10\end{array}$ & $\begin{array}{l}12 \\
10\end{array}$ & $\begin{array}{l}40.0 \\
33.3\end{array}$ & $\begin{array}{l}0 \\
0\end{array}$ & $\begin{array}{l}0.0 \\
0.0\end{array}$ & $\begin{array}{l}0 \\
0\end{array}$ & $\begin{array}{l}0.0 \\
0.0\end{array}$ & $\begin{array}{l}2 \\
4\end{array}$ & $\begin{array}{c}6.7 \\
13.3\end{array}$ & $\begin{array}{l}14 \\
10\end{array}$ & $\begin{array}{l}46.7 \\
33.3\end{array}$ & \\
\hline \multirow{6}{*}{$\begin{array}{l}\text { Education } \\
\text { - High } \\
\text { - Secondary } \\
\text { - Basic } \\
\text { - Read and write } \\
\text { - Non educated }\end{array}$} & & & & & & & & & & & & & \multirow{6}{*}{$0.03^{\prime \prime}$} \\
\hline & 0 & 0.0 & 3 & 10.0 & 0 & 0.0 & 0 & 0.0 & 0 & 0.0 & 1 & 1.3 & \\
\hline & 4 & 13.3 & 2 & 6.7 & 0 & 0.0 & 0 & 0.0 & 1 & 1.3 & 4 & 13.3 & \\
\hline & 0 & 0.0 & 2 & 6.7 & 0 & 0.0 & 0 & 0.0 & 0 & 0.0 & 2 & 6.7 & \\
\hline & 2 & 6.7 & 1 & 1.3 & 0 & 0.0 & 0 & 0.0 & 1 & 1.3 & 7 & 23.3 & \\
\hline & 2 & 6.7 & 13 & 43.4 & 0 & 0.0 & 0 & 0.0 & 4 & 13.3 & 10 & 33.3 & \\
\hline \multirow{4}{*}{$\begin{array}{l}\text { Occupation } \\
\text { - Office } \\
\text { - Manual } \\
\text { - Not working }\end{array}$} & & & & & & & & & & & & & \multirow{4}{*}{$0.38^{\mathrm{Ns}}$} \\
\hline & 0 & 0.0 & 5 & 16.7 & 0 & 0.0 & 0 & 0.0 & 0 & 0.0 & 1 & 1.3 & \\
\hline & 5 & 16.7 & 10 & 33.3 & 0 & 0.0 & 0 & 0.0 & 2 & 6.7 & 14 & 46.7 & \\
\hline & 3 & 10 & 7 & 23.3 & 0 & 0.0 & 0 & 0.0 & 4 & 13.3 & 9 & 30.0 & \\
\hline \multirow{4}{*}{$\begin{array}{l}\text { Marital status } \\
\text { - Single } \\
\text { - Married } \\
\text { - Widow }\end{array}$} & & & & & & & & & & & & & \multirow{4}{*}{$0.09^{\mathrm{Ns}}$} \\
\hline & 0 & 0.0 & 0 & 0.0 & 0 & 0.0 & 0 & 0.0 & 0 & 0.0 & 3 & 10.0 & \\
\hline & 8 & 26.7 & 17 & 56.7 & 0 & 0.0 & 0 & 0.0 & 4 & 13.3 & 18 & 30.0 & \\
\hline & 0 & 0.0 & 5 & 16.7 & 0 & 0.0 & 0.0 & 0.0 & 2 & 6.7 & 3 & 10. & \\
\hline \multirow{3}{*}{$\begin{array}{l}\text { Residence } \\
\text { - Urban } \\
\text { - Rural }\end{array}$} & & & & & & & & & & & & & \multirow{3}{*}{$0.32^{\mathrm{Ns}}$} \\
\hline & $\begin{array}{l}3 \\
5\end{array}$ & $\begin{array}{l}10.0 \\
167\end{array}$ & $\begin{array}{c}13 \\
9\end{array}$ & $\begin{array}{l}43.4 \\
30.0\end{array}$ & $\begin{array}{l}0 \\
0\end{array}$ & $\begin{array}{l}0.0 \\
0.0\end{array}$ & $\begin{array}{l}0 \\
0\end{array}$ & $\begin{array}{l}0.0 \\
0.0\end{array}$ & $\begin{array}{l}2 \\
8\end{array}$ & $\begin{array}{r}6.7 \\
267\end{array}$ & $\begin{array}{c}4 \\
16\end{array}$ & $\begin{array}{l}13.3 \\
53.3\end{array}$ & \\
\hline & & & & & & & & & & & & & \\
\hline
\end{tabular}

Person Chi-Square test and Independent samples test

**: highly significant $(P<0.01)$ 
Table (1): Comparison between study and control groups regarding demographic data: shows that the mean age of both study and control groups was $54.80 \pm 8.93$ and $48.23 \pm 13.72$ respectively, the highest percentage of them was male, non-educated, manual worker, married, from rural area, and smokers. No statistically significant difference was found between both groups regarding demographic data exception age.

Table (2): Comparison between study and control groups regarding medical data: presents that the highest percentage of both study and control groups have stage II renal cell carcinoma (46.7\%). Also, hypertension was the most common medical comorbidities $(23.3 \%)$. No statistically significant difference was found between both groups regarding medical data.

Table (3): Frequency distribution of clinical presentations among study and control groups before $\mathrm{RN}$ : reveals that flank pain was the most frequent clinical presentations in both groups $(70 \%)$, followed by gross hematuria (46.7\%).

Table (4): Distribution of factors responsible for preoperative anxiety among patients (study and control groups): illustrates that highly statistical significant differences were found between study and control groups regarding fear of result of operation and fear of death $(\mathrm{P}<0.01)$. Statistical significant difference was found between study and control groups regarding fear of postoperative pain and waiting for operation $(\mathrm{P}<0.05)$. Both study and control groups did not experience any fear regarding concern about family, fear for one's life, need of blood transfusion, financial loss, fear of unknown and harm from doctor/ nurse mistake.

Figure (1): Comparison between the study and the control groups regarding preoperative anxiety: presents that statistically significant difference was found between the study and control groups as regarding preoperative anxiety $(\mathrm{P}<0.01)$.

Table (5): Relation between demographic data and preoperative anxiety among the studies sample: illustrates that there were statistical significant relations between age, level of educational and preoperative anxiety $(\mathrm{P}<0.05, \mathrm{P}<0.01)$ respectively.

\section{Discussion}

Radical nephrectomy remains the most commonly performed procedure for the excision of RCC. Urologic patients undergoing $\mathrm{RN}$ require preoperative assessments and planning prior to intervention. Failure to properly assess the preoperative needs of these patients can potentially result in increased intra-operative and post-operative morbidity. It is important to understand the physiologic considerations in patients undergoing radical nephrectomy and when a patient is being considered for surgery, it is imperative to have a detailed discussion among the surgeon, anesthetist, patient, and nursing to optimize a satisfactory postoperative outcome (Nikhil et al., 2014 \& Tran et al., 2017)

Regarding demographic characteristics; the present study showed that; the mean age of the study and control groups was fifty one years. These findings are supported by (Chung et al., 2016) who mentioned that the mean age of the patients undergoing radical nephrectomy was fifty-seven years. This also was in concordance to the study by (Krebs et al., 2014) who reported that the mean age for the patient in the same situation was fifty- sex years. Also, (Gao et al., 2018) reported that, RCC are very common old group (4075) or old - old group.

Our study showed that slightly more than half of the study and control group patients were male. This result agreed with (Qureshi et al., 2015) who reported in their study that more than half of patients undergoing RN were male. According to (Zhang et al., 2018) more than two thirds of patients undergoing RN were male.

Regarding level of education, half of patients in the study group and approximately the same in the control group were non educated. This study finding was in agreement with (Tan et al., 2012) who revealed that the majority of patients were having low education.

In relation to marital status the present study revealed that the majority of patients were married. This agrees with (Tan et al., 2012) who reported that the majority of patients undergoing were married. This can be attributed to the more incidences of renal tumors in the fifth and sixth decades of age.

As regards to the residence, results from data collected in this study revealed that the majority of study and control groups were from in rural areas. these finding was supported by (Chang et al., 2018) who reported that highest percentage of patients lived in rural area. On the contrary (Tan et al., 2019) reported that that the majority of patients were from urban area. This difference is mostly due to the nature of area and population served by our hospital.

Regarding cigarette smoking it remains a highly prevalent habit in Egypt (Fouda et al., 2018). Smoking is the most consistent risk factor for RCC; however, the impact of smoking on survival in patients with RCC remains understudied. In the present, more than half of patients were smokers. These finding are consistent with (Zabor et al., 2016) who reported that majority of the studied 
patients were smokers. On the contrary (Ehdaie et al., 2014) told that more of the studied patients were nonsmokers.

Regarding medical data; presents that the highest percentage of both study and control groups have stage II renal cell carcinoma. Also, hypertension was the most common medical comorbidities. No statistically significant difference was found between both groups regarding medical data. (Kopp et al., 2014) agreed with these findings as they reported that hypertension was found in majority of patients. Also, (Khan et al., 2019) who reported that most of the patients undergoing RN had stage II of RCC.

Clinically, patients with renal cell carcinoma are reported asymptomatic or nonspecific symptoms such as fatigue, malaise and vague symptoms, and specific symptoms as flank pain and hematuria. This result agreed with (Laguna et al., 2014) who reported that more than half of patients presented with hematuria and more than one third with flank pain.

Regarding preoperative anxiety; there are various responsible factors for preoperative anxiety in patients undergoing RN. In this study, fear of complications were the main factors responsible for preoperative anxiety in both study and control groups. Fear of operation result and fear of death were seen more. (Mulugeta et al., 2018) were in the same line with the current study as they reported that the most common factors responsible for preoperative anxiety were fear of complications, concern about family, fear of postoperative pain and fear of death.

The present study proved that a significant decrease in the level of anxiety was found among the study group in comparison to the control group ones from the researcher point of view this may be due to the effect of the nursing teaching guidelines and the importance of preoperative education for the study patients undergoing RN. In the present study the demographic characteristics that were significantly associated with preoperative anxiety were age and educational level. Where the level of anxiety decreased with increasing level of education. This was parallel to other studies findings (Erkilic et al., 2017, Mulugeta et al., 2018, Abdelmowla et al., 2019) reported that the prevalence of preoperative anxiety was high. The levels of preoperative anxiety significantly reduce associated with preoperative education provision.

\section{Conclusion}

Where study group patient who received the nursing teaching guidelines showed a significant decrease in preoperative anxiety.

\section{Recommendation}

- Preoperative anxiety assessment should be incorporated into a routine nursing practice and every patient should be provided with preoperative information before surgery.

- Emphasize the importance to avoid emotional stress and stressful situations to avoid many complications that may develop from it.

- Establishment of health care educational center in the urology department to educate patients about necessary instructions regarding their conditions using booklet, illustrated pamphlets, models and videotapes for each patient especially those who cannot read and write.

- Replication of the study on a larger probability sample acquired from different geographical areas in Egypt to figure out the main aspects of these conditions and improve health.

\section{References}

1. Abdelmowla, R., Shahat, A., Ghanem H., \& Abdalla, M., (2019): The Importance of Verbal and Written Instructions for Patients Undergoing Pressure-Flow Urodynamic Studies, ARC Journal of Nursing and Healthcare 5 (3), 8-16

2. Alper, I., \& Yüksel, E., (2016): Comparison of Acute and Chronic Pain after Open Nephrectomy versus Laparoscopic Nephrectomy: A Prospective Clinical Trial. 95(16), 3433.

3. Chang, Y., Chang, S., Liu, C., Lin, P., Yu, K., Pang, S., \& Shao, I., (2018): Demographic characteristics and complications of open and minimally invasive surgeries for renal cell carcinoma: a population-based case-control study in Taiwan. Therapeutics and clinical risk management, 14, 1235

4. Chung, S., Huang, C., Wu, S., Lin, H., Huang, C., \& Kao, L., (2016): Nephrectomy type was not associated with a subsequent risk of coronary heart disease: A population-based study. PloS one, 11(9), 1-8.

5. Draeger, D., Sievert, K., \& Hakenberg, O., (2018): Analysis of psychosocial stress factors in patients with renal cancer. Therapeutic advances in urology, 10(6), 175-182.

6. Ehdaie, B., Furberg, H., Zabor, E., Hakimi, A., \& Russo, P., (2014): Comprehensive assessment of the impact of cigarette smoking on survival of clear cell kidney cancer. The Journal of urology, 191(3), 597-602.

7. Erkilic, E., Kesimci, E., Soykut, C., Doger, C., Gumus, T., \& Kanbak, O., (2017): Factors 
associated with preoperative anxiety levels of Turkish surgical patients: from a single center in Ankara. Patient preference and adherence, 11, 291.

8. Fouda, S., Kelany, M., Moustafa, N., Abushouk, A., Hassane, A., Sleem, A., \& Bassiony, M., (2018): Tobacco smoking in Egypt: a scoping literature review of its epidemiology and control measures. EMHJEastern Mediterranean Health Journal, 24(02), 198-215.

9. Gao, X., Hu, L., Pan, Y., \& Zheng, L., (2018): Surgical outcomes of nephrectomy for elderly patients with renal cell carcinoma. Pakistan journal of medical sciences, 34(2), 288.

10. Harris, R., Oake, K., Hawkins, R., Jones, R., Powles, T., \& Montgomery, D., (2015): Patient needs in advanced Renal Cell Carcinoma: What are patients' priorities and how well are we meeting them? Patient Experience Journal, 2(2), 142-152.

11. Karabulut, N., \& Cetinkaya, F., (2011): The impact on the level of anxiety and pain of the training before operation given to adult patients. Surgical Science, 2(06), 303.

12. Khan, M., Patel, R., Jain, N., Balakrishnan, A., \& Venkataraman, M., (2019): Prospective analysis of laparoscopic versus open radical nephrectomy for renal tumours more than $7 \mathrm{~cm}$. Journal of minimal access surgery, 15(1), 14.

13. Komolafe, C., Csernus, M., \& Fülöp, E., (2015): Patients' anxiety during the perioperative care from the point of view of the nursing staff and patients. Kontakt, 17(2), 8088.

14. Kopp, R., Mehrazin, R., Palazzi, K., Liss, M., Jabaji, R., Mirheydar, H., \& Patterson, A., (2014): Survival outcomes after radical and partial nephrectomy for clinical $\mathrm{T} 2$ renal tumours categorised by RENAL nephrometry score. BJU international, 114(5), 708-718.

15. Krebs, R., Andreoni, C., \& Ortiz, V., (2014): Impact of radical and partial nephrectomy on renal function in patients with renal cancer. Urologia internationalis, 92(4), 449-454.

16. Laguna, M., Algaba, F., Cadeddu, J., Clayman, R., Gill, I., Gueglio, G., \& Van Poppel, H., (2014): Current patterns of presentation and treatment of renal masses: a clinical research office of the endourological society prospective study. Journal of endourology, 28(7), 861-870.

17. Mirza, K., Taxy, J., \& Antic, T., (2016): Radical nephrectomy for renal cell carcinoma: its contemporary role related to histologic type, tumor size, and nodal status: a retrospective study. American journal of clinical pathology, 145(6), 837-842.

18. Mulugeta, H., Ayana, M., Sintayehu, M., Dessie, G., \& Zewdu, T., (2018): Preoperative anxiety and associated factors among adult surgical patients in Debre Markos and Felege Hiwot referral hospitals, Northwest Ethiopia. BMC anesthesiology, 18(1), 155.

19. Nikhil, C., Poon, A., Gowrie-Mohan, S., Lane, T., Boustead, G., Hanbury, D., \& Adshead, J., (2014): The physiologic and anesthetic considerations in elderly patients undergoing robotic renal surgery. Reviews in urology, 16(1), 1.

20. Palese, A., Tomietto, M., Suhonen, R., Efstathiou, G., Tsangari, H., Merkouris, A., \& Karlou, C., (2011): Surgical patient satisfaction as an outcome of nurses' caring behaviors: a descriptive and correlational study in six European countries. 43(4), 341-350.

21. Pastore, A., Maruccia, S., Mir, A., Palleschi, G., Carbone, A., Bellonch, N., \& Palou, J., (2017): Psychological distress in patients undergoing surgery for urological cancer: A prospective single centre cross-sectional study. European Urology Supplements, 16(3), e1172e1174.

22. Pinto, A., Faiz, O., Davis, R., Almoudaris, A., \& Vincent, C., (2016): Surgical complications and their impact on patients' psychosocial wellbeing: a systematic review and meta-analysis. BMJ open, 6(2), 1-23.

23. Qureshi, H., Shehzad, A., Mohsin, R., Sultan, G., Laghari, R., Mubarak, M., \& Rizvi, S., (2015): Impact of radical nephrectomy on renal functional outcome in patients with no other comorbidity as determined by $24-\mathrm{h}$ urinary creatinine clearance. African Journal of Urology, 21(4), 210-213.

24. Ryamukuru, D., (2017): Assessment of preoperative anxiety for patients awaiting surgery at UTHK. University of Rwanda, 1-51.

25. Spielberger, C., Gorsuch, R., Lushene, R., Vagg, P., \& Jacobs, G., (1983): Manual for the state-trait anxiety inventory (Palo Alto, CA, Consulting Psychologists Press). Inc.

26. Tan, H., Norton, E., Ye, Z., Hafez, K., Gore, J., \& Miller, D., (2012): Long-term survival following partial vs radical nephrectomy among older patients with early-stage kidney cancer. Jama, 307(15), 1629-1635.

27. Tan, W., Berg, S., Cole, A., Krimphove, M., Marchese, M., Lipsitz, S., \& Kibel, A., (2019): Comparing long-term outcomes following 
radical and partial nephrectomy for cT1 renal cell carcinoma in young and healthy individuals. JNCI Cancer Spectrum, 3(1), 1-8.

28. Tran, V., Lyon, M., Ha, S., Warncke, J., Cost, N., \& Wilson, S., (2017): Review of management options for localized renal cell carcinoma. Minim Invasive Surg Oncol, 1, 85102.

29. Vukovic, N., and Dinic, L., (2018): Enhanced recovery after surgery protocols in major urologic surgery. Frontiers in Medicine, 5, 93.

30. Zabor, E., Furberg, H., Mashni, J., Lee, B., Jaimes, E., \& Russo, P., (2016): Factors associated with recovery of renal function following radical nephrectomy for kidney neoplasms. Clinical Journal of the American Society of Nephrology, 11(1), 101-107.

31. Zhang, M., Zhao, Z., Duan, X., Deng, T., Cai, C., Wu, W., \& Zeng, G., (2018): Partial versus radical nephrectomy for T1b-2N0M0 renal tumors: A propensity score matching study based on the SEER database. PloS one, 13(2), 111. 